\title{
Shedding Frequency in Cavitation Erosion Evolution Tracking
}

Markku Ylönenª, Jean-Pierre Franc ${ }^{b}$, Juha Miettinenc, Pentti Saarenrinned, Marc Fivele

aDoctoral student, Tampere University, Faculty of Engineering and Natural Sciences, Korkeakoulunkatu 6, 33720 Tampere, Finland. E-mail: markku.ylonen@tuni.fi ${ }^{b}$ CNRS Research Professor, Univ. Grenoble Alpes, CNRS, Grenoble INP, LEGI, 38000 Grenoble, France. E-mail: jean-pierre.franc@legi.cnrs.fr 'Docent, Tampere University, Faculty of Engineering and Natural Sciences, Korkeakoulunkatu 6, 33720 Tampere, Finland. E-mail: juha.miettinen@tuni.fi dEmeritus Professor, Tampere University, Faculty of Engineering and Natural Sciences, Korkeakoulunkatu 6, 33720 Tampere, Finland. E-mail: pentti.saarenrinne@tuni.fi eCNRS Research Professor, Univ. Grenoble Alpes, CNRS, Grenoble INP, SIMaP, 38000 Grenoble, France. E-mail: marc.fivel@simap.grenoble-inp.fr

\section{Abstract}

Cavitation erosion is a concern for most hydro machine operation. An especially damaging type of cavitation is the cloud cavitation. This type has a growth-collapse cycle in which a group of vapor bubbles grows together in a low-pressure region, to collapse almost simultaneously when the pressure recovers. Measuring the frequency of these collapse events is possible by acoustic emission ( $A E)$, as demonstrated in this study, in which a cavitation tunnel is utilized to create cloud cavitation on a sample surface. These samples were fitted with $A E$ sensors, and the initially high frequency $A E$ signal was demodulated to detect the relatively low frequency cloud cavitation shedding. When the cavitation number is increased, AE detects the changes in this frequency correctly, confirmed by comparing the results to video analysis and to simulations by other authors. Additionally, the frequency increases when cavitation erosion progresses, thus it provides means to track the erosion stage. The presented method can be used in detecting the transition from cloud to sheet cavitation when the cavitation number is increased, and in tracking erosion evolution in the cavitation tunnel. The method could probably be extended to hydro machine monitoring, as this type of cloud cavitation is common in hydrofoils. 
Keywords: Cavitation erosion; cloud cavitation; shedding frequency; acoustic emission.

\section{Introduction}

Cavitation is the growth and collapse of vapor bubbles in a static liquid or a liquid flow due to decrease and the subsequent recovery of static pressure. The collapse of these bubbles potentially damages adjacent surfaces, individual bubbles causing typically local and micrometer-scale damage. However, as the bubbles are quite numerous and the duration of exposure to cavitation is generally long, damage tends to accumulate and reach macroscopic levels. This causes serious harm to the structural integrity and flow performance of hydro machines, pipes, and valves. All damage is difficult to avoid, when searching for the maximum performance and utility. Additionally, the extent of erosion is not usually well known during operation, as it is difficult to monitor. Cavitation often occurs inside flow channels, where continuous visual observation may be difficult and costly, if not impossible.

This study focuses on cloud cavitation, which is a common type of cavitation observed in various hydraulic equipment and in particular in hydrofoils. Cloud cavitation is a highly eroding type of cavitation. A cloud of vapor bubbles forms in the leading edge of a hydrofoil, due to an increase in flow velocity, which leads to the decrease in static pressure. It travels downstream the foil, and collapses towards the trailing edge, if the static pressure recovers sufficiently along the foil. The cloud is not static; it grows and collapses periodically due to its process of growth, formation of a re-entrant flow, shedding, and collapse. The shedding frequency depends on flow velocity, surface quality, overall pressure, temperature and surrounding liquid quality. (Brennen et al. 2000; Franc and Michel 2005; Nishimura et al. 2014; Gnanaskandan and Mahesh 2016; Hsiao et al. 2017) 
The shedding phenomenon greatly depends on cavitation number. Smaller cavitation numbers lead to larger scale cavitation structures (Peng et al. 2016). The cavitation structures strongly depend on hydrofoil or wall geometries and the angle of attack (Kravtsova et al. 2014). Additionally, the laminar-turbulent transition plays a great role in the formation of vortices (Chen et al. 2018). The changes in shedding frequency due to varying cavitation number were observed also in this study.

The current study had two main approaches: 1 ) detecting the change in cloud shedding frequency via acoustic emission $(\mathrm{AE})$ and confirming the results through comparison to highspeed video analysis and to simulation results by other authors, and 2) tracking cavitation erosion evolution through shedding frequency measured via AE. Material samples that were fitted with AE sensors were eroded in a cavitation tunnel that creates cloud cavitation. Changes in the cloud cavitation shedding frequency is not a common way to monitor cavitation erosion, but in the case of a well-developed cavitation cloud, such as in this study, it proved to be an efficient method. A series of erosion tests were conducted, along with a 'ramp test' to study the effects of cavitation number. The ramp test was a series of tests with varying cavitation number and with a non-eroded sample.

Acoustic emission is defined as elastic waves traveling in a solid material (Holroyd 2000; Grosse 2008). These elastic waves are the result of energy released in the material. The energy release may be due to internal stresses, external impacts or surface contacts. As an AE source, cavitation represents external impacts, resulting from bubble collapses near a boundary. $A E$ has a wide frequency band, resulting from wave front reflections in the solid medium, the range being typically from $100 \mathrm{kHz}$ to $1 \mathrm{MHz}$ (Pollock 1986). Two main types of sensors exist: the resonance type and the broadband sensor, with the main difference being the damping 
properties in sensor structure. They are placed on a solid surface that has a good transfer path to the signal source. $A E$ is a tempting method in monitoring cavitation, as it is non-intrusive and sensors can be placed in the machine structure. The main challenge is the interpretation of the results, as the signal voltage is not easily transformed into any physical parameter, such as pressure or acceleration. (Ohtsu et al. 2016; Achenbach 1975; Inaba 2016; Trampe Broch 1984)

AE has been used in cavitation detection and erosion intensity evaluation, but not in erosion evolution tracking, as far as the authors know of. AE detects the incipience of cavitation efficiently (Alfayez and Mba 2005; Neill et al. 1997), enabling early detection of possibly harmful cavitation, for example in journal bearing lubrication films (Poddar and Tandon 2016). Cavitation that has damage potential occurs typically close to surfaces; bubbles collapsing in the free flow cause pressure shocks, but do not damage the surfaces that are far away. Therefore, properly placed AE sensors detect only the collapses occurring near boundaries and leading to impacts towards the material surface (van Rijsbergen et al. 2012). The nondamaging collapse events happening far away from the surface lead to no significant sensor response.

The presented approach begins by demonstrating the applicability of $A E$ in shedding frequency monitoring by comparing the detected frequencies to those calculated from a video analysis and to those from simulations by Gavaises et al. (2015). Then, the method is used in tracking how the frequency changes when the material surface erodes, thus modifying the flow field in the cavitation test section. The presented method provides an effective way to monitor changes in cloud shedding frequency, due to erosion evolution or changes in flow velocity, provided the cloud shedding phenomenon is pronounced enough. 


\section{Experimental program}

In the experiments, two different stainless steels were eroded in a high-speed cavitation tunnel, while recording $\mathrm{AE}$ signal. A 'ramp test' of varying cavitation number was also performed. The authors of Gavaises et al. (2015) provided their video recordings from the cavitation tunnel, which were reanalyzed to complement the $A E$ results concerning the cavitation cloud shedding frequencies.

\subsection{Testing equipment and procedure}

The cavitation test rig was the PREVERO cavitation tunnel (PREVERO 2018). The data acquisition board was a PAC PCI-2, with two sensors: the PAC R15D resonance type sensor, and the PAC D9203b broadband sensor, both used with a PAC 2/4/6 preamplifier. The preamplifier had a $100 \mathrm{kHz}$ to $400 \mathrm{kHz}$ band-pass analogue filter for the R15D and a $100 \mathrm{kHz}$ to $1200 \mathrm{kHz}$ one for the D9203b. The sampling rate was $5 \mathrm{MHz}$ per channel. The dataacquisition software was PAC AEWin ${ }^{\mathrm{TM}}$ and the signals were further analyzed in Matlab ${ }^{\mathrm{TM}}$.

The cavitation tunnel consists of a water loop, a test section, a pump, and a pressurizing tank, along with pressure, temperature, and flow meters. The tunnel is pressurized by an external nitrogen tank connected to the downstream tank of the tunnel. Below the downstream tank is the pump that has a frequency regulator to control rotation speed, and therefore the upstream pressure and flow velocity of the tunnel. In the same level as the downstream tank is the test section where cavitation occurs. The pressure loss in the test section is the dominating pressure loss source in the tunnel. The cavitation tunnel was operated with a constant cavitation number of 0.87 for the mass loss tests, and with a varying cavitation number for the ramp test. The cavitation number $\sigma$ in the tunnel is defined as (Franc et al. 2012): 
$\sigma=\frac{P_{d}-P_{v}}{P_{u}-P_{d}}$

where $P_{u}$ is the tunnel upstream pressure or the pressure before the test section, $P_{d}$ is the tunnel downstream pressure or the pressure after the test section, and $P_{v}$ is the saturated vapor pressure. A constant cavitation number, regardless of overall pressure level, leads to equal radial position of the cavitation closure on the material sample (Gavaises et al. 2015). The pressure difference $P_{u}-P_{d}$ is linked to the overall flow velocity, which is a significant contributor to cavitation aggressiveness. If the upstream pressure is increased, the cavitation number decreases; therefore, to retain a constant cavitation number, the downstream pressure needs to be increased. The rise in overall pressure level corresponds to increase in cavitation aggressiveness (Franc et al. 2012).

The samples are $100 \mathrm{~mm}$ diameter and $20 \mathrm{~mm}$ thick cylinders. They have one flat, uniform, and initially mirror polished face for cavitation erosion tests. The other face, or the back of the sample, is used for fixing the sample to the sample holder by a screw in the center. The test section is an axisymmetric, radially diverging channel, one channel wall being the material sample. The nozzle directs a water jet in the middle of the sample, where the water flow stagnates and diverges radially. The flow cross section area drops to $62.5 \%$ of the original, thus dropping the static pressure under the critical value for cavitation inception. As the flow further diverges and the static pressure increases due to increasing cross section area, cavitation closes, leading to erosion of the sample and flow channel surface. Cavitation occurs parallel to the sample surface. Fig. 1 illustrates the test section and the cavitation inception and closure, along with a photograph of an eroded material sample fixed to the sample holder. Areas A-D correspond to areas later used in defining the different areas in the video analysis. A is the nozzle area, where the flow stagnates, B is the area of the growth of the 
cavitation cloud, $\mathrm{C}$ is the cavitation closure area, between $19 \mathrm{~mm}$ and $32 \mathrm{~mm}$ radial position from the centerline if $\sigma \approx 0.9$, and $D$ is the area where cavitation is no longer observed.

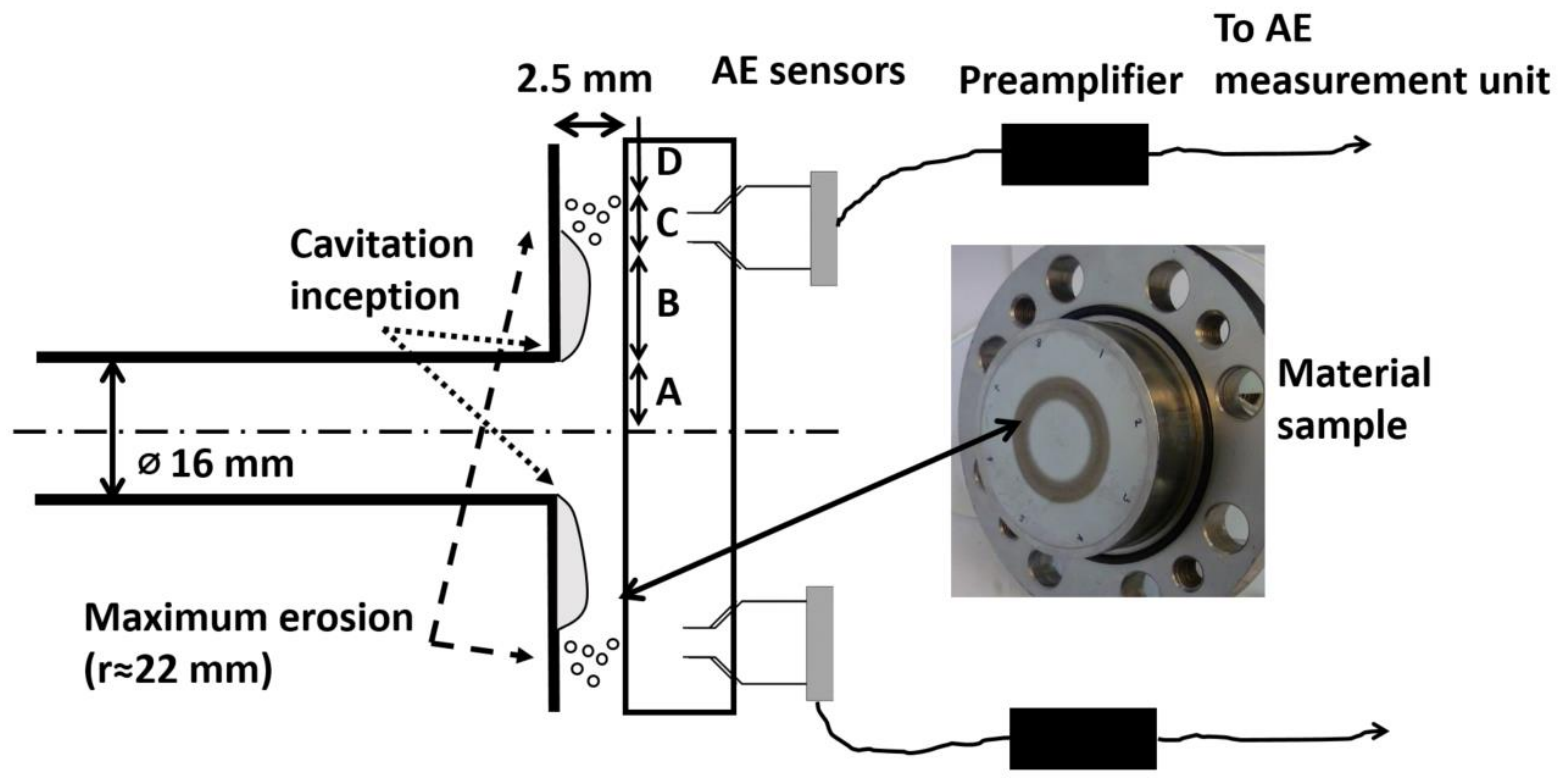

Fig. 1. Test section schematics of the cavitation tunnel and the AE setup and a photograph of an eroded material sample fixed to the sample holder. Areas A-D are later used in the video analysis in Figs. 3-7.

The two eroded samples were made of martensitic stainless steel and they were named stainless steel 1 and stainless steel 2 . They were subjected to cavitation erosion for about 65 hours. Both of them were specimens from Francis turbine runner blades. The material properties did not have any significance in the current approach, as the analysis concentrated on the shedding frequency analysis, which was assumed a flow related phenomenon. This study utilizes the material volume loss test results by Ylönen et al. (2018), where the material properties were also thoroughly examined. Therefore, the volume loss curves used for comparison later in this study are the same ones already presented.

Volume loss and erosion depth were calculated from surface profiles that were measured with a Taylor-Hobson Form Talysurf 50 contact profilometer. The sample profile was measured along eight equally divided azimuthal angles of the sample. The measurement was 
from the center towards the edge of the circular sample surface. The surface profiles were mostly measured in five-hour intervals, excluding the beginning of the tests, where the interval was either thirty minutes or two hours. The upstream pressure was $4 \mathrm{MPa}$ and the downstream pressure 1.9 MPa for the volume loss tests, corresponding to maximum tunnel aggressiveness.

In the ramp test, the cavitation number was increased from well-developed cavitation up to the point where cavitation disappears, and further on to non-cavitating flow. The cavitation number was increased gradually by decreasing the upstream pressure, while keeping the downstream pressure constant. The upstream pressure in the beginning was $4 \mathrm{MPa}$ and it was decreased in steps down to $2.3 \mathrm{MPa}$, with the downstream pressure remaining at 1.9 MPa. Cavitation disappeared at $\sigma \approx 2.8$, the observation based on audible noise from the tunnel and on the significant drop in AE levels.

\subsection{Video analysis}

The videos were a courtesy of Gavaises et al. (2015), who already analyzed them to find cavitation structures and shedding frequencies. They had a vast campaign of capturing different operating points, but only five videos that corresponded to the operation points in the ramp tests were chosen for the present study. They were filmed from the direction where the sample would normally be, the sample replaced by a Perspex window. The videos covered only a partial area of the tunnel test section. The filming procedure is further explained by Gavaises et al. (2015). In this study, these videos were reanalyzed with a slightly different approach; the video analysis was an FFT analysis of the gray level of each pixel.

The videos were recorded with a frame rate of 77000 frames per second. The visualized area was $34 \times 16 \mathrm{~mm}^{2}$, the pixel size was $132 \times 125 \mu \mathrm{m}^{2}$, the image size was $128 \times 256$ pixels and 
the video was directly recorded in grayscale. Their lengths and operation points in the cavitation tunnel are presented in Table 1.

\begin{tabular}{l|l|l|l|l} 
Video ID & $\begin{array}{l}\text { Cavitation } \\
\text { number } \sigma\end{array}$ & $\begin{array}{l}\text { Upstream } \\
\text { pressure (MPa) }\end{array}$ & $\begin{array}{l}\text { Downstream } \\
\text { pressure (MPa) }\end{array}$ & Length (frames) \\
\hline C27 & 1.897 & 2.909 & 1.905 & 1738 \\
C28 & 1.515 & 3.166 & 1.907 & 1495 \\
C29 & 1.159 & 3.562 & 1.912 & 1356 \\
C30 & 0.908 & 4.027 & 1.916 & 1331 \\
C31 & 0.811 & 4.273 & 1.913 & 2895
\end{tabular}

Table 1. Video lengths and cavitation tunnel operation points.

For each pixel in the video, the grayscale value was captured, values picked frame by frame. An example of a video frame is presented in Fig. 2.

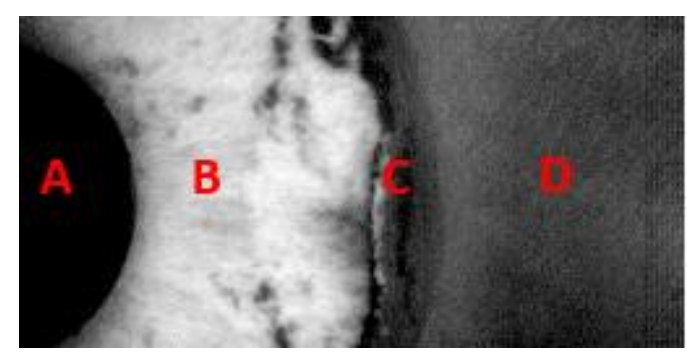

Fig. 2. An individual frame from video C30. Markers: A) Flow stagnation from the nozzle, covered for filming, B) Cavitation cloud growth, C) Cavitation closure and D) No cavitation. The areas (A-D) are also presented in Fig. 1.

The most interesting areas in the images are B and C. Area A was masked for the video analysis and area $D$ has no cavitation. The grayscale value as a function of time was obtained for each individual pixel and for all videos, and they were analyzed via FFT. The frequency bin size is limited and given by:

$f_{b i n}=F_{S} * \frac{L}{2}$

where $f_{\text {bin }}$ is the frequency bin size, $F_{s}$ is frame rate and $L$ is the video length in frames. The videos were relatively short, C30 being the shortest one. The frequency bin size for C30 was $51.5 \mathrm{~Hz}$. Therefore, the peak frequency values have an error margin of $+/-26 \mathrm{~Hz}$. This was 
considered not too high, as the shedding frequencies were above $1600 \mathrm{~Hz}$, so the relative error was in the order of $2 \%$.

The frequency maps for the analyzed videos with different values of the cavitation number $\sigma$ are presented in Figs. 3-7. The zero and close to zero frequencies in the A and D regions correspond to the masked nozzle area and the downstream area with no cavitation. In Fig. 7, most of the area, including regions $B$ and $C$, had no measurable frequencies. In all videos, several individual pixels were found having a more than $10 \mathrm{kHz}$ frequency. These were interpreted as local anomalies, and therefore they were excluded. The dominating frequency, where it is well definable, was assumed to correspond to cavitation cloud shedding frequency.

Fig. 3 and Fig. 4 present the peak frequency maps for relatively low cavitation number $(0.8<$ $\sigma<1.0)$

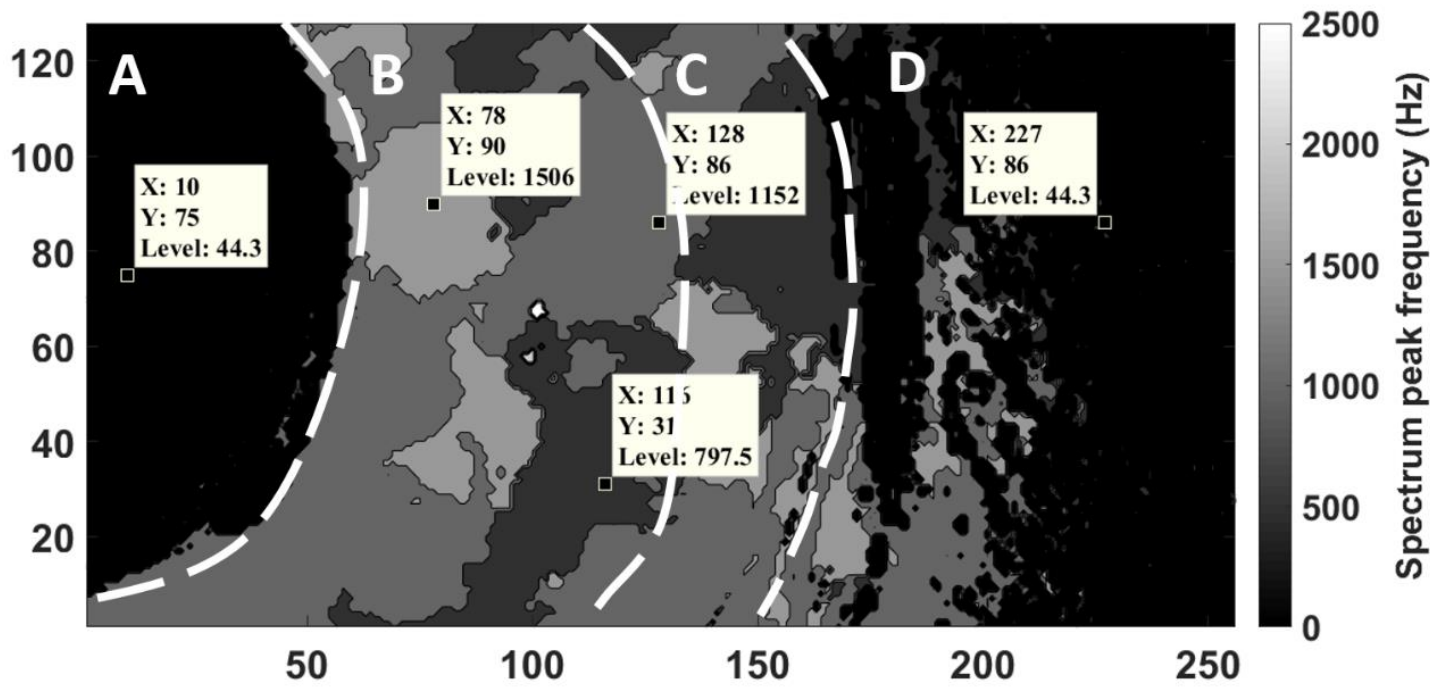

Fig. 3. Frequency map of $\sigma=0.811$. The $X$ and $Y$-axes represent the pixel coordinates in the video. 


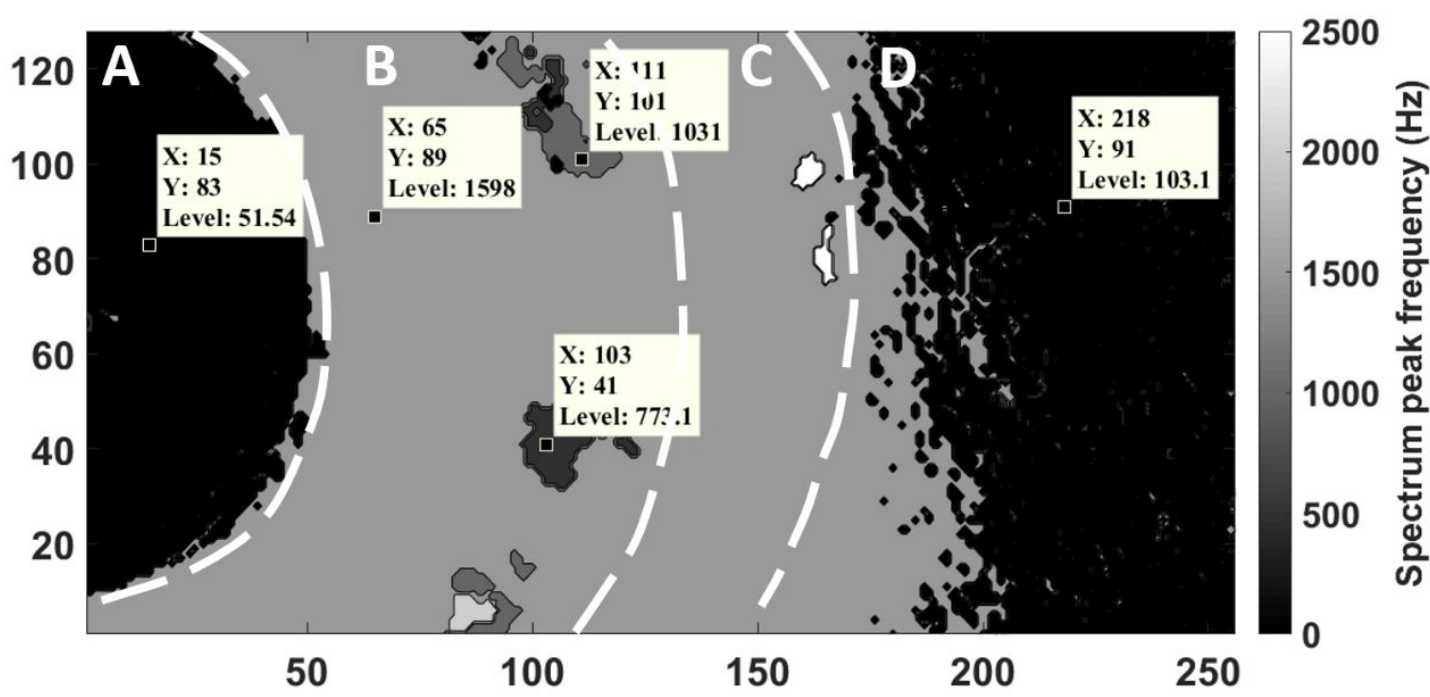

Fig. 4. Frequency map of $\sigma=0.908$. The $X$ and $Y$-axes represent the pixel coordinates in the video.

The dominating frequency for $\sigma=0.811$ in Fig. 3 cannot be well defined. Three major frequency zones were identified: $798 \mathrm{~Hz}, 1152 \mathrm{~Hz}$ and $1506 \mathrm{~Hz}$, the largest in area being 1152 $\mathrm{Hz}$. The $1506 \mathrm{~Hz}$ would be in line with the other results, and it is the one presented in the comparison of methods later on. Longer videos might have made the results more representable, but none was available. When $\sigma=0.908$ in Fig. 4 , the dominating frequency is clearly $1598 \mathrm{~Hz}$. Fig. 5 and Fig 6. present the peak frequency maps for higher cavitation numbers, where cloud shedding is still observed $(1.1<\sigma<1.6)$. 


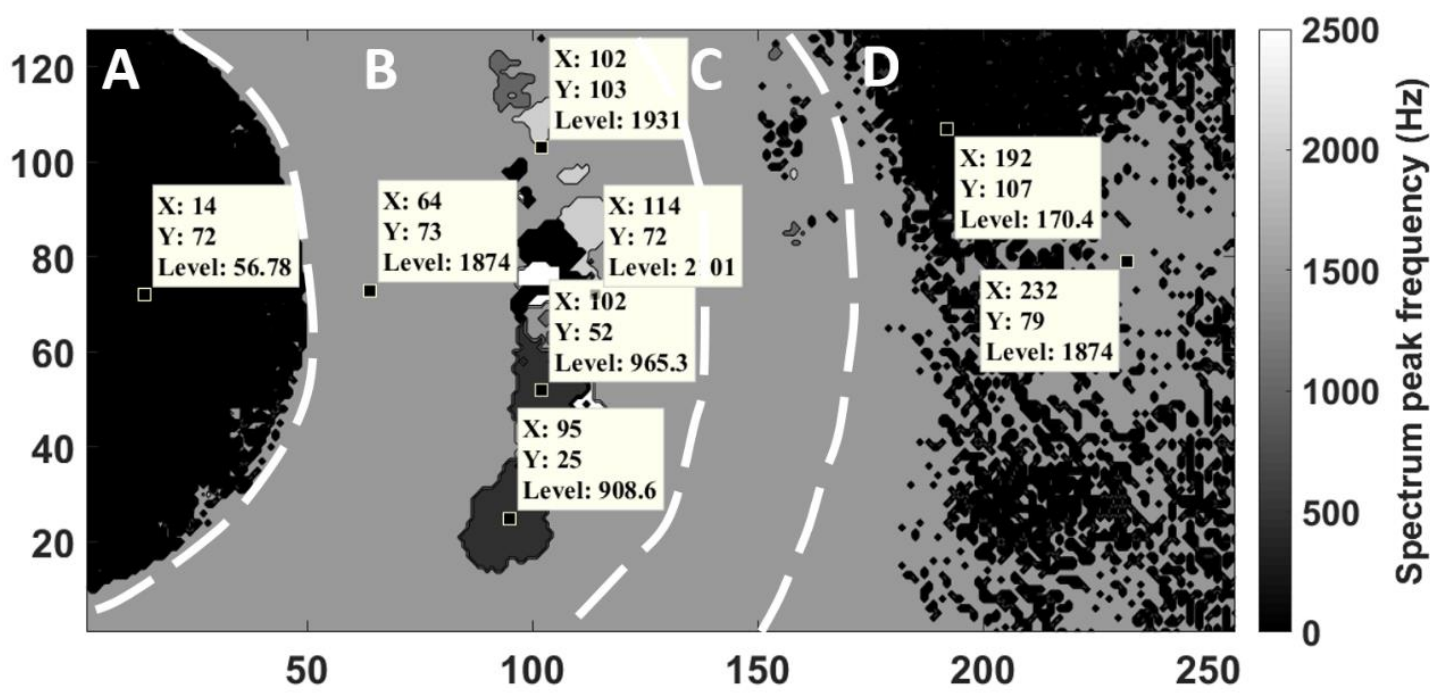

Fig. 5. Frequency map of $\sigma=1.159$. The $X$ and $Y$-axes represent the pixel coordinates in the video.

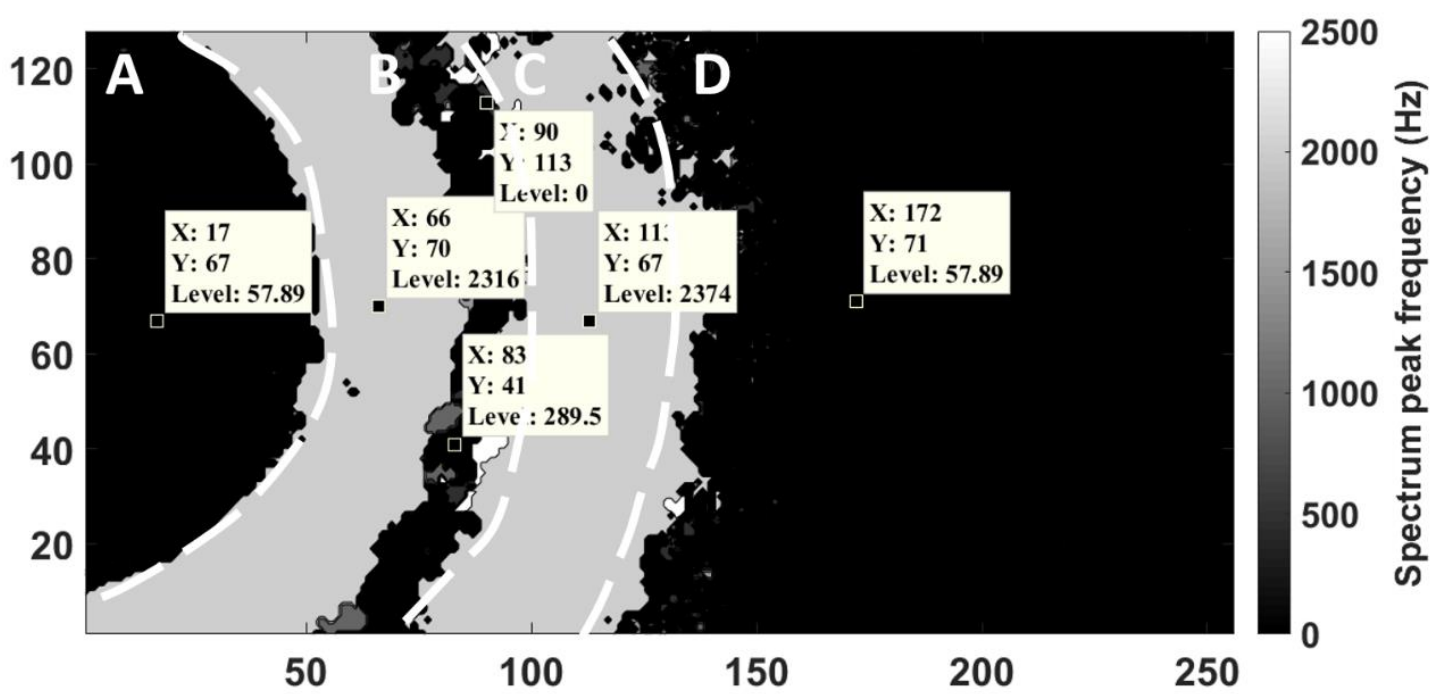

Fig. 6. Frequency map of $\sigma=1.515$. The $X$ and $Y$-axes represent the pixel coordinates in the video.

When $\sigma=1.159$ in Fig. 5, the dominating frequency of $1874 \mathrm{~Hz}$ can be well defined. When $\sigma$ $=1.515$ in Fig. 6, two different dominating frequencies were observed: $2316 \mathrm{~Hz}$ and $2374 \mathrm{~Hz}$, and between them, a narrow band of multiple different frequencies. It is notable that the two dominating frequencies represent neighboring frequency bins. Therefore, they probably represent the same frequency that happens to be close to the average of these two frequencies. As the frequency resolution is limiting the observations, $2374 \mathrm{~Hz}$ was picked as the cloud shedding frequency, as it is situated in the area of cavitation closure (region C). In 
Fig. 7 , the cavitation number is so high that presumably the shedding phenomenon ceases to exist, at least in a significant scale. Using the same color map as for the other figures, Fig. 7 is almost completely black, as only several frequency peaks were detected.

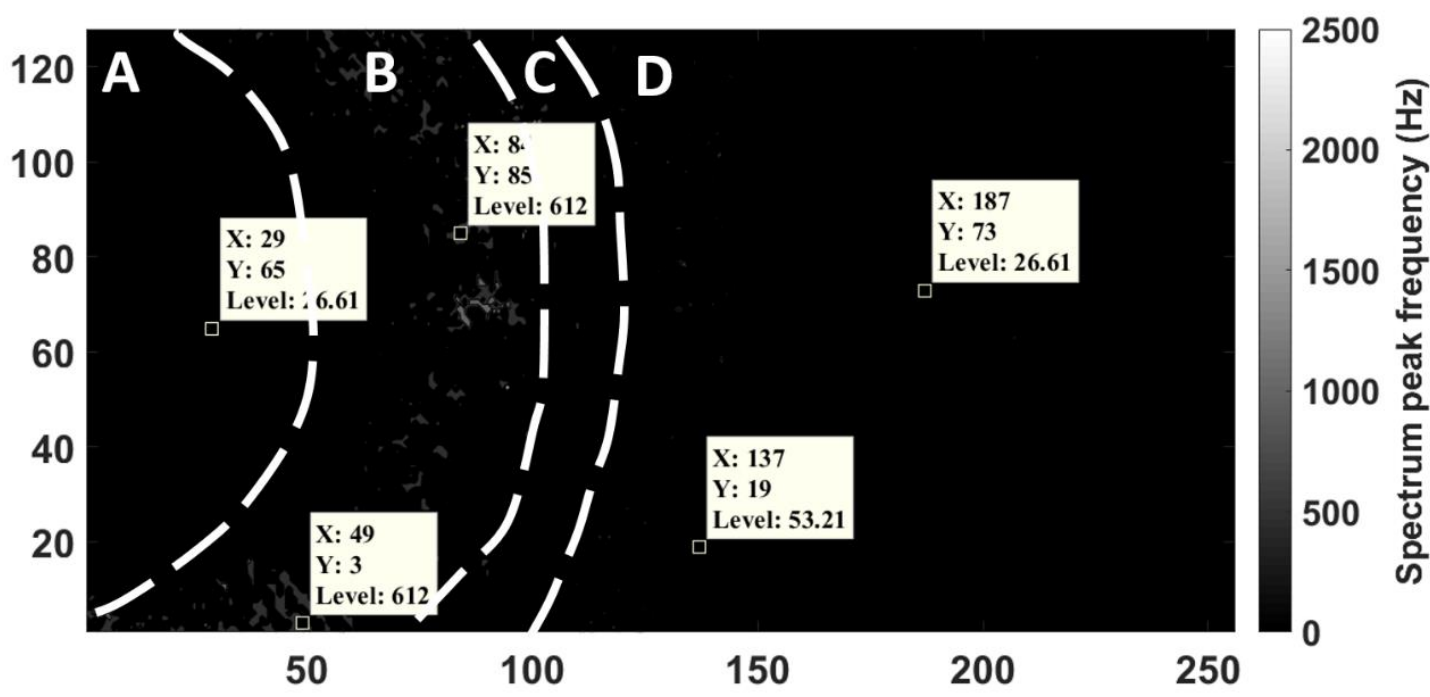

Fig. 7. Frequency map of $\sigma=1.897$. The $X$ and $Y$-axes represent the pixel coordinates in the video.

When $\sigma$ increases, the cavitation cloud shedding becomes less and less pronounced. A similar disappearance of a dominating frequency was found in the AE signal analysis. Therefore, it seems that the cloud shedding phenomenon is well observable only with low cavitation numbers, as also noted by Gavaises et al. (2015). This suggests a transition from could cavitation to sheet cavitation, when the cavitation number is increased (Pelz et al. 2017).

\section{Acoustic emission signal processing}

The initial goal in this study was to track changes in AE energy, RMS-value, peak frequencies, and amplitudes when cavitation erosion evolves. This was tempting, as the acquisition system tracked these parameters by default. The values did fluctuate and their levels changed during the cavitation tests, but no proper trend was found in the current study. Therefore, they were left out from further analysis in this paper. 
Raw voltage signal was also recorded during the erosion, in roughly one-hour intervals with a five-minute acquisition time, except for the first sixty minutes that were recorded in full. The sampling frequency was $5 \mathrm{MHz}$ for both sensors. He and Shen (2012) stated that the signal average energy would have a good correlation with the mass loss rate in their tests using an ASTM G32-10 (ASTM 2010) vibratory cavitation testing apparatus. However, the present investigation did not confirm their observation, since no clear correlation between the signal average energy and the mass loss rate could be found.

To validate that $A E$ properly captures cavitation events, the AE signals from several different cavitation numbers are presented in Fig. 8 . The exact voltages that are induced by cavitation are not important in this study, but comparing amplitudes in Fig. 8 clearly shows that when cavitation disappears, the AE levels drop over a hundred-fold compared to the fullydeveloped cavitation. The operating condition in Fig. 8D has practically no cavitation, but dropping the cavitation number about 0.2 would lead to the inception of cavitation. Fig. $8 \mathrm{~A}$ and Fig. $8 \mathrm{~B}$ represent conditions where cloud shedding was observed in the video and $\mathrm{AE}$ analysis, while Fig. 8C corresponds to cavitation with assumedly no pronounced cloud growthcollapse cycle. 

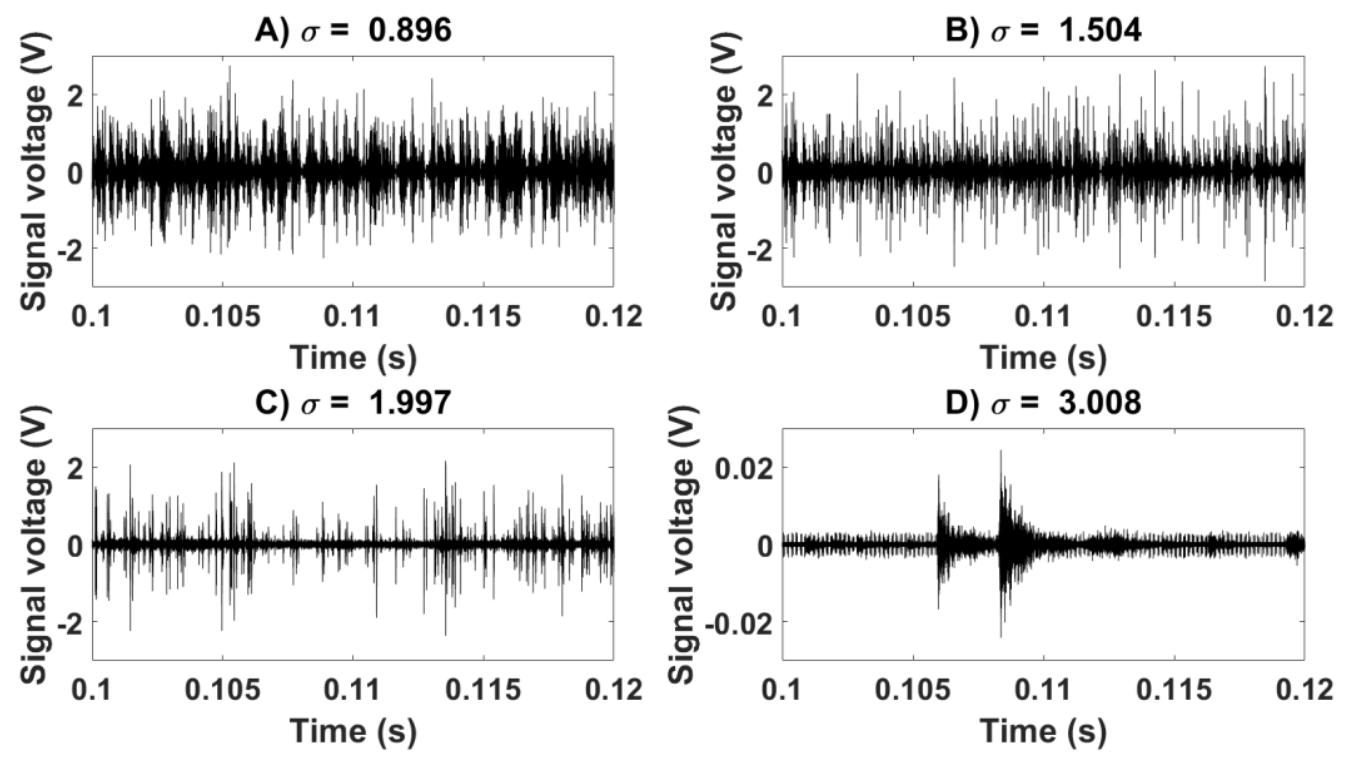

Fig. 8. AE signal voltage levels for varying cavitation numbers. A) and B) represent fully developed cavitation with cloud shedding, C) represents cavitation that is assumed to be sheet cavitation, and $D$ ) represents a cavitation number where cavitation no longer occurs.

The signal amplitudes might correlate with the strength of cavitation erosion, but the current approach was based on signal frequencies. The frequency responses of the AE sensors are rather non-linear. This means that the frequency content of the signals is highly colored with the sensor frequency response. Therefore, the initially observed maximum frequency from raw voltage signals is usually the peak value of the sensor frequency response.

It was expected that the cavitation cloud shedding frequency would be at several thousand $\mathrm{Hz}$. The AE signals were band-pass filtered with a lower limit frequency of $100 \mathrm{kHz}$, so it could be expected that the low frequency shedding could not be detected. However, it is detectable using signal demodulation techniques. Events, such as cavitation cloud shedding, occurring at lower frequencies, have a high frequency content inside them that is not lost in the filtering process. At time intervals corresponding to the shedding frequency, a rise in AE activity is observed, the signal frequencies inside the shedding event being in the sensor frequency range. 
Berry (1997a) utilized a waveform enveloping and demodulation technique to promote low frequency content in signals. The method presents a way to for example, track bearing failures (Berry 1997b). It works with any signal that has low frequency content that is interesting, but that is not properly detectable using direct spectral analysis and when the high frequency content acts as a modulated carrier signal. The demodulation process in this study was essentially similar to that of Berry, although signal enveloping was performed according to the description by Marple Jr (1999). The procedure, modified from the description by the previous authors to suit the needs of the current signals, was the following:

1) $\mathrm{AE}$ was measured with band-pass filtering from $100 \mathrm{kHz}$ to $400 \mathrm{kHz}$ or $100 \mathrm{kHz}$ to 1200 $\mathrm{kHz}$, depending on the sensor. The signal contained also the high frequency content of the repeating shedding events. The low frequencies of the shedding were "hidden" in the high frequency content.

2) The signal average was subtracted, with the average value saved for later corrections

3) A fast Fourier transform (FFT) was performed to the zero-averaged signal. The average was initially close to zero, as AE signals generally are close to symmetric around zero volts.

4) The frequency domain one-sided discrete-time analytic signal was calculated, with the mathematical formulation in Marple Jr (1999)

5) The discrete-time analytic signal magnitude was calculated via inverse FFT (IFFT). This corresponds to the creation of an envelope of the original signal. The original signal average was added to the enveloped signal. The modulated carrier signal is lost, thus the low frequency contents were promoted.

6) A low-pass filter was applied, with the decimation of the signal. The low-pass filter was an eighth order Chebyshev Type I low-pass filter, the default of the Matlab ${ }^{\mathrm{TM}}$ function 
'decimate'. The decimation resampled the signal with a lower frequency, to reduce sample sizes, in addition to the low-pass filtering. The original sampling rate was not required, as the high frequency content was filtered out at this point.

7) The filtered signals were transformed to frequency domain by Welch's method. The window size was 5,000 samples and the window overlapping was $50 \%$.

8) Two frequencies with the highest amplitudes were detected and saved for further analysis

The demodulation process effectively promotes the low frequency content in the signal; the low frequency content exists also in the non-demodulated frequency data, but it is usually lost because the high frequency content is dominating. Fig. 9 presents the FFT analysis of both an original and a demodulated signal. The shedding frequency is observable from the original data, but not reliably, as the high frequency amplitudes are about ten orders of magnitude larger.

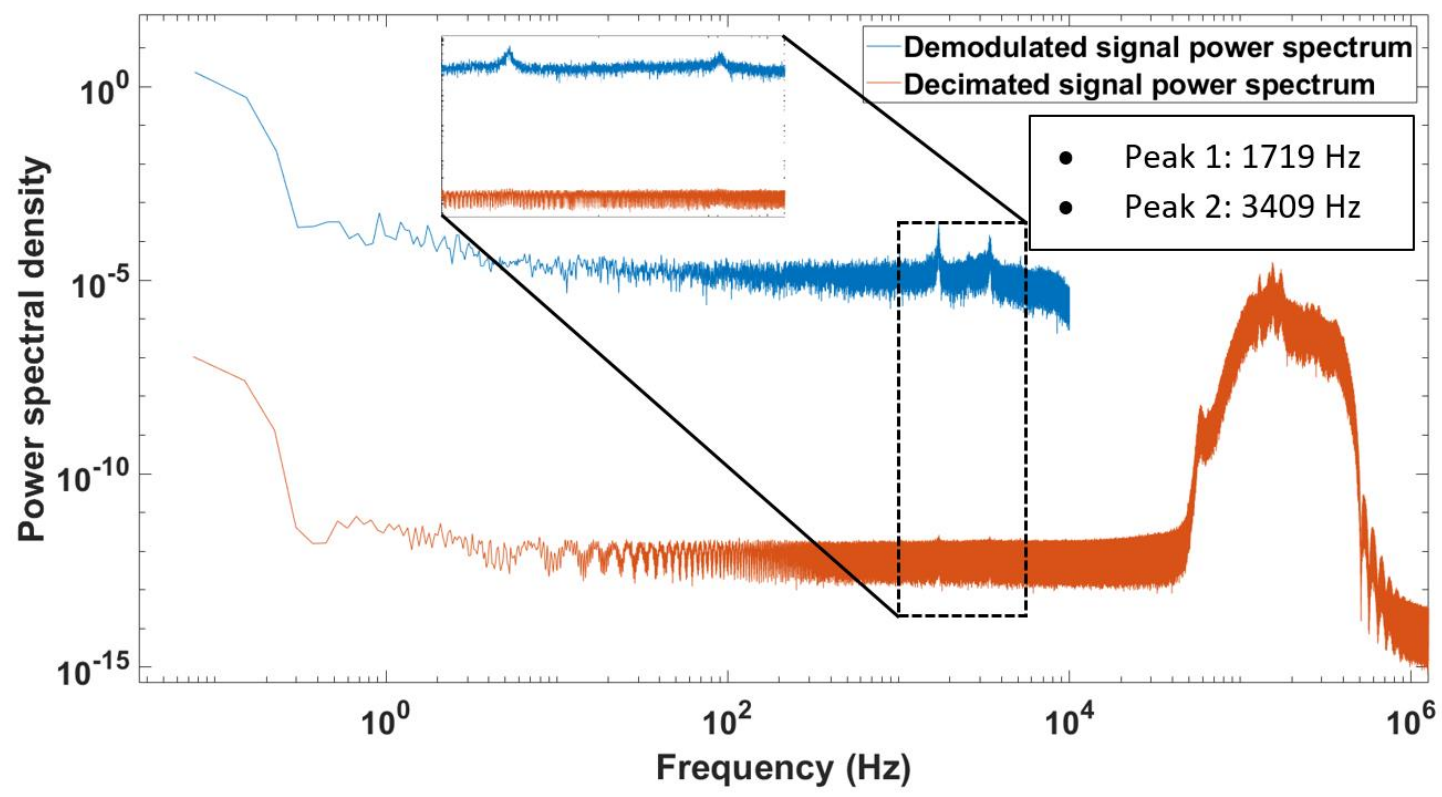

Fig. 9. The demodulated signal power spectral density compared to that of the original signal.

\section{Results}




\subsection{Shedding frequency in the ramp tests}

The ramp test went through well-developed cavitation to no cavitation state of the cavitation tunnel. All the $A E$ waveforms were demodulated, for both sensors. Typically, two distinguishable peak frequencies were detected, as in Fig. 9. The peak frequencies as a function of cavitation number are presented in Fig. 10. The higher frequency peaks were interpreted as the first harmonic frequency of the shedding frequency, as its frequency was always two times the lower one, and it was excluded from further analysis. Each marker in Fig. 10, represents a thirty-second interval. Six of these were measured and analyzed for each cavitation number. The first harmonic is also marked in Fig. 10, to highlight that it follows the same trend as the actual shedding frequency.

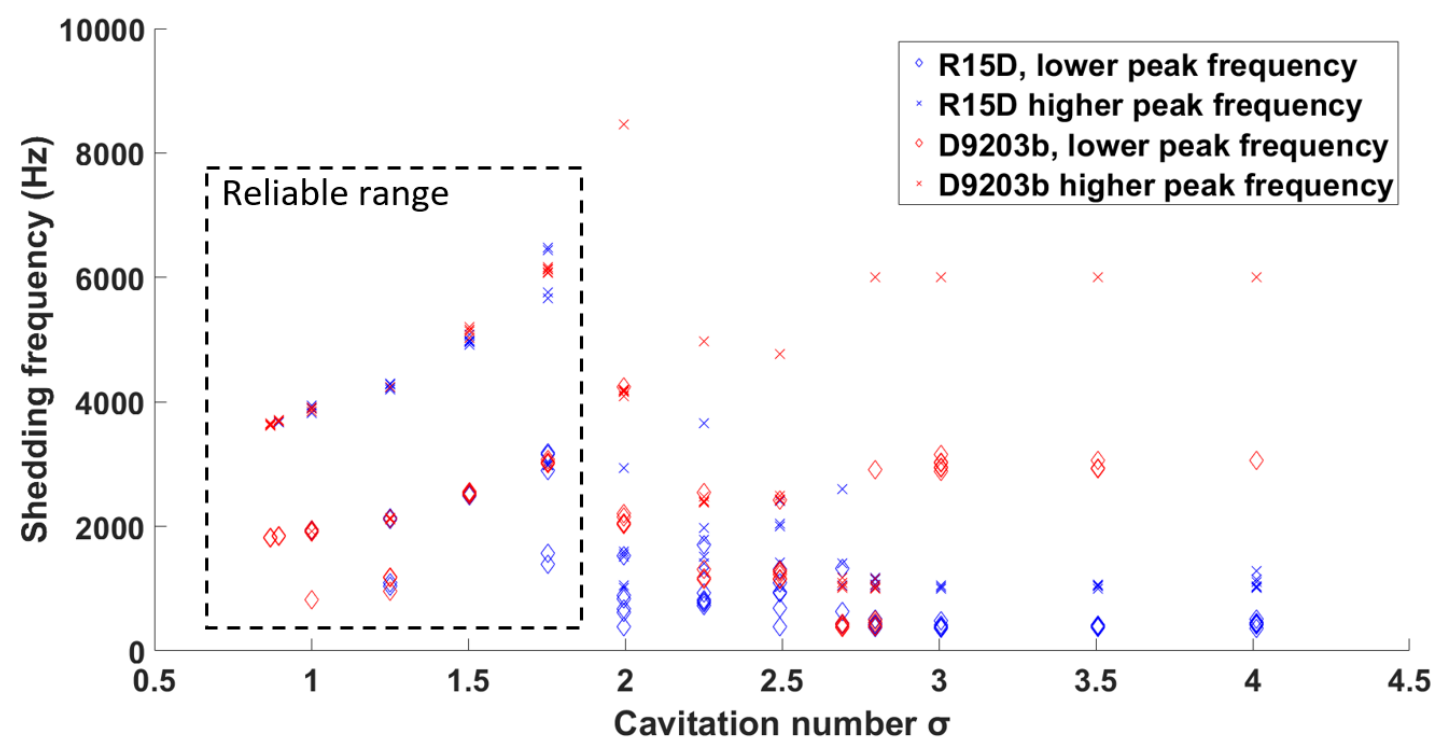

Fig. 10. Cavitation shedding frequency measured by $A E$ as a function of cavitation number.

Both the resonance type sensor (R15D) and the broadband sensor (D9203b) detect the same frequency, in most cases. The linear trend in frequencies is lost after $\sigma>1.75$, although already at $\sigma=1.75$, the frequencies do not anymore follow that precisely the trend. The consistency is lost between $\sigma \approx 1.5$ and $\sigma \approx 1.8$ also in the video analysis. This suggests that 
the shedding phenomenon does not anymore exist, with cavitation numbers above a certain limit.

\subsection{Shedding frequency: comparison between $A E$ and videos}

This section aims to evaluate the AE capability in capturing the correct shedding frequency.

The AE frequencies deduced from the demodulation process were compared to the shedding frequencies found from the video analysis and the simulation results by Gavaises et al. (2015), who simulated the erosion via computational fluid dynamics (CFD), using a hybrid RANS/LES model. The most important result of theirs in the scope of this study was the relation between cavitation number and shedding frequency, reprinted with permission in Fig. 11.
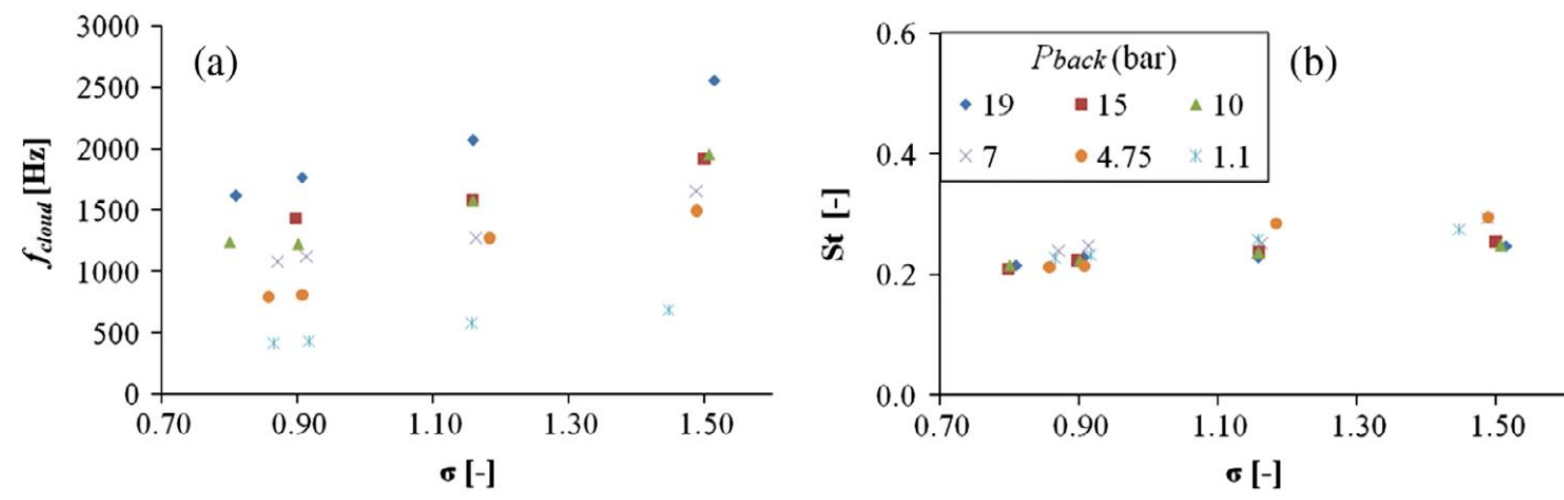

Fig. 11. Simulated shedding frequency as a function of cavitation number in the cavitation tunnel by Gavaises et al. (2015). The Strouhal number St was defined as the ratio of shedding frequency multiplied by the gap size of $2.5 \mathrm{~mm}$ and the mean flow velocity at the $25 \mathrm{~mm}$ radius of the diverging channel. Reprinted from International Journal of Multiphase Flow, Vol. 68, M. Gavaises, F. Villa, P. Koukouvinis, M. Marengo, J. Franc, Visualisation and les simulation of cavitation cloud formation and collapse in an axisymmetric geometry, Fig. 15, Copyright 0301-9322/C 2014 Elsevier Ltd, with permission from Elsevier.

Gavaises et al. (2015) simulated multiple downstream pressures $\left(P_{\text {back }}\right)$. The shedding frequency clearly increases with increasing cavitation number, at all pressure levels. A lower downstream pressure corresponds to a lower shedding frequency. In this study, the analysis was limited to $1.9 \mathrm{MPa}$, or 19 bar downstream pressure, so only those values were kept for 
Fig. 12, which compares all the three different methods to determine the shedding frequency when the cavitation number is varied.

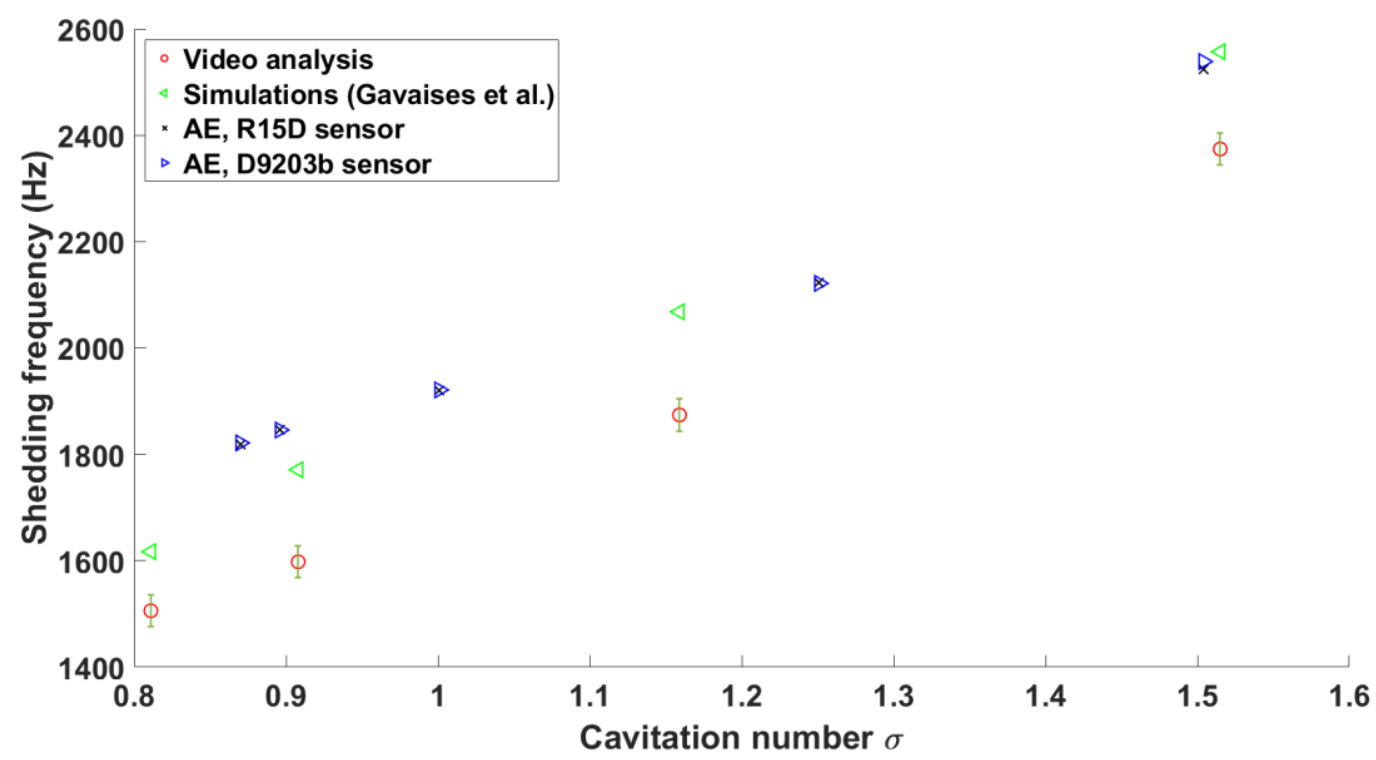

Fig. 12. Comparison of the results by Gavaises et al. (2015), the video analysis, and the AE measurements, regarding the relation between shedding frequency and cavitation number.

The error bars in the video analysis are the result of the FFT bin size. The maximum bin size was $51.5 \mathrm{~Hz}$, so the maximum error was rounded to $+/-30 \mathrm{~Hz}$. The AE result standard deviation was always less than $12 \mathrm{~Hz}$ between the thirty-second intervals, therefore it was not feasible to visualize in the figure. Fig. 12 shows that all the three different methods are in good agreement. It is thus concluded that the shedding frequency can be captured reliably and with sufficient accuracy from AE measurements, using the presented demodulation processing technique. The result is valid at least for the cavitation tunnel.

\subsection{Shedding frequency from AE and cavitation erosion}

The previous results demonstrated that AE captures the proper shedding frequency, so a similar analysis was carried out for cavitation erosion tests, in order to highlight a potential drift in shedding frequency with changing surface geometry. The material samples were eroded from a virgin non-eroded surface to approximately $400-\mu \mathrm{m}$ maximum erosion depth. 
Both samples took about 65 hours to reach this state. Stainless steel 2 eroded more in volume and average erosion depth than stainless steel 1; therefore, it had an inferior resistance to cavitation, analyzed in more detail by Ylönen et al. (2018).

AE was measured in one- to two-hour intervals, excluding the beginning of the tests, where it was measured continuously. The waveforms were collected for five minutes at each interval, and the five-minute waveform was split into thirty-second sections that were individually analyzed following the demodulation algorithm. Fig. 13A presents the evolution of the shedding frequency, for both samples and both AE sensors. The volume losses and erosion depths for both samples are presented in Fig. 13B. Fig. 13C presents the shedding frequency as a function of volume loss, while Fig. 13D presents the shedding frequency as a function of erosion depth. The erosion depth was the maximum profile depth at each measurement point.
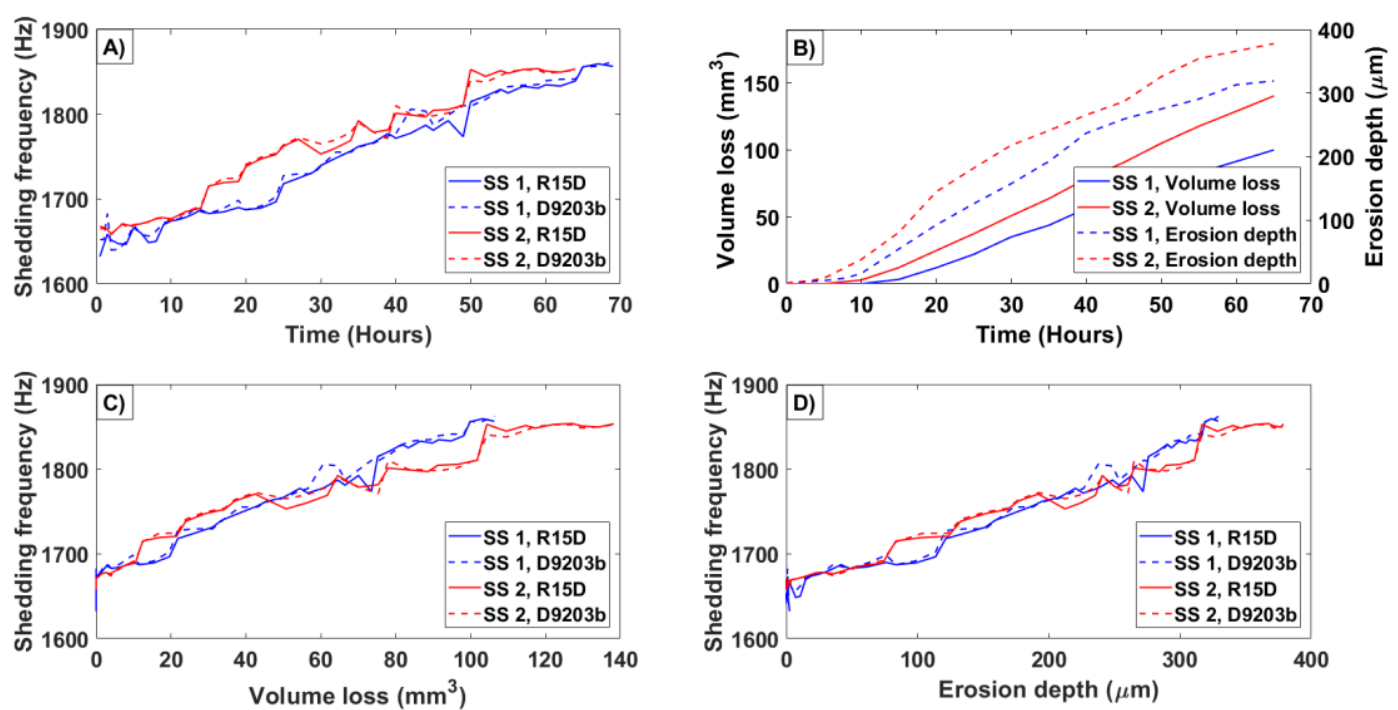

Fig. 13. Cavitation shedding frequency as a function of erosion time, combined with volume loss and erosion depth. Cavitation number $\sigma=0.87$.

It is notable that the shedding frequency, the volume loss and the erosion depth of stainless steel 2 (SS2) begins to increase faster than those of stainless steel 1 (SS1), after about 12 hours 
of erosion, or roughly after the cavitation incubation period. In the incubation period, the surface deformation rate is slower than in the advanced periods, meaning that the increase in roughness is smaller. After 48 hours of erosion, the slopes of the shedding frequency curves begin to decrease, reaching possibly a constant value. This suggests that the changes in roughness might have little effect when the surface is highly damaged. It could be interpreted so that after a critical level of roughness, the shedding frequency becomes roughness independent. This is, however, not possible to confirm with this limited investigation.

When plotting the shedding frequency as a function of volume loss in Fig. $13 \mathrm{C}$ and erosion depth in Fig. 13D, the plots for the two different tests and materials overlap to a significant degree. This suggests that the shedding frequency is a parameter that is linked only to the surface deformation of the samples. The relation is linear at least between 0 to $100 \mathrm{~mm}^{3}$ volume loss, or between 0 to $350 \mu \mathrm{m}$ erosion depth. The shedding frequency tends to fluctuate, as seen in the plots, so the error in defining either volume loss or erosion depth from shedding frequency would be significant, although a rough estimate could be obtained.

When the material surface is degraded, the resulting surface roughness leads to modifications in the flow field and therefore in the shedding phenomenon. The increase in shedding frequency with the increase in roughness is consistent with the results by Hao et al. (2017). Their rough hydrofoil experiencing cloud cavitation had a $20 \mathrm{~Hz}$ shedding frequency, whereas for a smooth one in equal conditions, the frequency was $17 \mathrm{~Hz}$. They explained the difference by the rough surface having a more complex flow pattern, including initial and large-scale shedding, with different type of attachment to the surface. Contrary to those results and results in this study, Stutz (2003) found no effect of roughness in the case of a cavitation sheet. 
This suggests that a cavitation cloud is more sensitive than a cavitation sheet to changes in roughness.

The shedding frequency is a well definable parameter in cloud cavitation. Therefore, measuring its change in a hydro machine would give information about the state of cavitation erosion. Acoustic emission is a promising option for measuring the frequency, as the sensors can be placed outside the flow, the measurements being non-intrusive and non-destructive. As long as the cavitation number is constant between two separate measurements, an unchanged shedding frequency corresponds to no change in surface roughness or erosion evolution. However, for a shedding phenomenon to exist and to be detectable, cavitation has to be well developed, as pointed out in the cavitation number analysis.

\section{Conclusions}

The cavitation cloud shedding frequency was detected reliably with both the video analysis and $A E$, in cavitation tunnel tests. The frequencies were in good agreement with those simulated by Gavaises et al. (2015), for the same test channel geometry. The AE signal demodulation process proved to be efficient in promoting the shedding frequency that is significantly lower than the lower frequency limit of the AE acquisition system. The AE signals were band-pass filtered so that the lower frequency limit was $100 \mathrm{kHz}$, and the cavitation shedding frequency was between $1600 \mathrm{~Hz}$ and $1900 \mathrm{~Hz}$. The high frequency AE signal acted as a modulated carrier signal for the shedding phenomenon, so the low frequency content was not lost with the initial filtering process. Effectively, the shedding frequency remained in the signal, as the cavitation cloud growth-collapse process was periodical, with the collapse stage inducing high loads that were well detected by AE. 
The cavitation shedding phenomenon becomes less pronounced as the cavitation number $\sigma$ increases. The limit of no cavitation state in the tunnel was at $\sigma \approx 2.8$, but both the video analysis and the $\mathrm{AE}$ analysis begun to fail in finding a shedding frequency when $\sigma>1.5$. A potential explanation is provided by the research of Pelz et al. (2014) and Pelz et al. (2017), who studied the transition between a stationary sheet cavity and cloud cavitation. A sheet cavity does not have a periodical structure, so there is no definable shedding frequency. Additionally, a sheet cavity is much less erosive than cloud cavitation; therefore, it should induce less $A E$. If one would reliably measure the cloud shedding frequency in a hydro machine, the disappearance of the well-defined shedding would reveal the transition from cloud cavitation to stationary sheet cavitation, i.e. the transition from an erosive cavitation to a less erosive type of cavitation. This would require knowledge of the frequency range of the shedding phenomenon, obtained either from CFD or from experiments.

In addition to being able to detect the transition from cloud to sheet cavitation, AE use was demonstrated to be effective in erosion evolution tracking. As the increasing surface roughness leads to increase in the cloud shedding frequency (Hao et al. 2017), the shedding frequency increases while erosion progresses. The shedding frequency was about $1600 \mathrm{~Hz}$ when the samples were non-eroded, and about $1850 \mathrm{~Hz}$ when they were eroded to the practical maximum of the cavitation tunnel. This gradual increase of the frequency was linked to the volume loss and the erosion depth, which were parameters defining erosion evolution. The frequencies were virtually the same for a resonance type and a broadband type AE sensor that were used simultaneously during the tests. The frequencies were not significantly affected by the stainless steel quality of the samples, only by the stage of erosion. It is therefore assumed that the shedding frequency is a parameter related to surface geometry and the flow field. 
The presented method could be extended to hydro machine erosion stage monitoring, if either the machine experiences cloud cavitation, or the machine could be deliberately moved into a stage where cloud cavitation occurs, even if this stage is not in the normal operation region. No change in shedding frequency between measurements would correspond to no change in erosion evolution. The main advantage of using $A E$ to define the shedding frequency would be the ease of installation in an existing machine, with no intrusion to the flow, along with the possibility to continuously measure as long as needed.

\section{Acknowledgements}

The authors would like to thank Business Finland, Fortum Power and Heat Oy, Sandvik Mining and Construction Oy, Valtra Oy, Teollisuuden Voima Oyj, and Fortum Foundation for funding the research and for providing technical support. The authors would also like to thank Fabio Villa and Phoevos Koukouvinis for providing their cavitation shedding videos for reanalysis and for the discussion on how to interpret the results.

\section{References}

Achenbach, J. D., 1975. Wave Propagation in Elastic Solids, North Holland Publishing

Alfayez, L., and Mba, D., 2005. Detection of incipient cavitation and determination of the best efficiency point for centrifugal pumps using acoustic emission, Proc. Inst. Mech. Eng. Part E J. Process Mech. Eng., 219(4), 327-344.

ASTM International, 2010. ASTM G32-10, Standard Test Method for Cavitation Erosion Using Vibratory Apparatus.

Berry, J. E., 1997a. High Frequency Enveloping and Demodulation Techniques Using Instruments From Various Vendors, Technical Associates of Charlotte, P.C.

Berry, J. E., 1997b. Tracking of Rolling Element Bearing Failure Stages Using Both Vibration Signature Analysis as well as High Frequency Envelopping and Demodulation Special Techniques, Technical Associates of Charlotte, P.C.

Brennen, C., Colonius, Y., Wang, A., and Preston, A., 2000. Cloud Cavitation Phenomena, TwentySecond Symposium on Naval Hydrodynamics, 239-253. 
Chen, Y., Li, J., Gong, Z., Chen, X., and Lu, C., 2018. Large eddy simulation and investigation on the laminar-turbulent transition and turbulence-cavitation interaction in the cavitating flow around hydrofoil, International Journal of Multiphase Flow, In press.

Franc, J., and Michel, J., 2005. Fundamentals of Cavitation, Springer Science + Business Media, Inc.

Franc, J., Riondet, M., Karimi, A., and Chahine, G. L., 2012. Material and velocity effects on cavitation erosion pitting, Wear, 274-275, 248-259.

Gavaises, M., Villa, F., Koukouvinis, P., Marengo, M., and Franc, J., 2015. Visualisation and les simulation of cavitation cloud formation and collapse in an axisymmetric geometry, International Journal of Multiphase Flow, 68, 14-26.

Gnanaskandan, A., and Mahesh, K., 2016. Large Eddy Simulation of the transition from sheet to cloud cavitation over a wedge, International Journal of Multiphase Flow, 83, 86-102.

Grosse, C., 2008. Acoustic Emission Testing. Springer Berlin Heidelberg, Heidelberg, Germany.

Hao, J., Zhang, M., and Huang, X., 2017. The influence of surface roughness on cloud cavitation flow around hydrofoils, Acta Mechanica Sinica, 34(1), 10-21.

He, Y., and Shen, Z., 2012. Experimental Research on Cavitation Erosion Detection Based on Acoustic Emission Technique, 30th European Conference on Acoustic Emission Testing \& 7th International Conference on Acoustic Emission, 1-8.

Holroyd, T., 2000. Acoustic Emission and Ultrasonics Handbook (Coxmoor's Machine \& System Condition Monitoring), Coxmoor Publishing Co., Moreton-in-Marsh, United Kingdom.

Hsiao, C., Ma, J., and Chahine, G. L., 2017. Multiscale tow-phase flow modeling of sheet and cloud cavitation, International Journal of Multiphase Flow, 90, 102-117.

Inaba, H., 2016. AE Sensor (AE Transducer), Practical Acoustic Emission Testing, The Japanese Society for Non-Destructive Inspection, 35-45.

Kravtsova, A. Y., Markovich, D. M., Pervunin, K. S., Timoshevskiy, M. V., and Hanjalić, K., 2014. Highspeed visualization and PIV measurements of cavitating flows around a semi-circular leading-edge flat plate and NACA0015 hydrofoil, International Journal of Multiphase Flow, 60, 119-134.

Marple Jr, L., 1999., Computing the discrete-time "analytic" signal via FFT, IEEE Transactions on Signal Processing, 47, 2600-2603.

Neill, G. D., Reuben, R. L., Sandford, P. M., Brown, E. R., and Steel, J. A., 1997. Detection of incipient cavitation in pumps using acoustic emission, Proceedings of the Institution of Mechanical Engineers, 211(4), 267-277.

Nishimura, S., Takakuwa, O., and Soyama, H., 2014. Effect of Nozzle Geometry on Aggressivity of Cavitating Jet for Cavitation Erosion Test and Applications, Advanced Experimental and Numerical Techniques for Cavitation Erosion Prediction, K. Kim, G. Chahine, J. Franc, and A. Karimi, eds., Springer Netherlands, Dordrecht, 283-302. 
Ohtsu, M., Enoki, M., Mizutani, Y., and Shigeishi, M., 2016. Principles of the Acoustic Emission (AE) Method and Signal Processing, Practical Acoustic Emission Testing, The Japanese Society for NonDestructive Inspection, 5-35.

Pelz, P., Keil, T., and Groß, T., 2017. The transition from sheet to cloud cavitation, J.Fluid Mech., 817, 439-454.

Pelz, P., Keil, T., and Ludwig, G., 2014. On the Kinematics of Sheet and Cloud Cavitation and Related Erosion, Fluid Mechanics and its Applications, 106, 221-237.

Peng, X. X., Ji, B., Cao, Y., Xu, L., Zhang, G., Luo, X., and Long, X., 2016. Combined experimental observation and numerical simulation of the cloud cavitation with U-type flow structures on hydrofoils, International Journal of Multiphase Flow, 79, 10-22.

Poddar, S., and Tandon, N., 2016. Detection of journal bearing vapour cavitation using vibration and acoustic emission techniques with the aid of oil film photography, Tribology International, 103, 95101.

Pollock, A.A., 1986. Classical Wave Theory in Practical AE Testing, Progress in Acoustic Emission III: proceedings of the 8th International Acoustic Emission Symposium, pp. 708-722.

PREVERO, 2018. The PREVERO cavitation erosion tunnel.

http://web.archive.org/web/20180327065723/http://www.legi.grenobleinp.fr/web/spip.php?article1265\&lang=fr $(25 / 10,2018)$.

Stutz, B., 2003. Influence of Roughness on the Two-Phase Flow Structure of Sheet Cavitation, Journal of Fluids Engineering, 125(4), 652-659.

Trampe Broch, J., 1984. Acoustic Emission, Mechanical vibration and shock measurements, Brüel \& Kjær, 212-236.

van Rijsbergen, M., Foeth, E., Fitzsimmons, P., and Boorsma, A., 2012. High-Speed Video Observations and Acoustic-Impact Measurements on a NACA 0015 Foil, 8th International Symposium on Cavitation (CAV 2012), 958-964.

Ylönen, M., Saarenrinne, P., Miettinen, J., Franc, J., Fivel, M., and Nyyssönen, T., 2018. Cavitation Erosion Resistance Assessment and Comparison of Three Francis Turbine Runner Materials, Materials Performance and Characterization, 7(5), 1107-1126.

\section{Figure Captions}

Fig. 1. Test section schematics of the cavitation tunnel and the AE setup and a photograph of an eroded material sample fixed to the sample holder. Areas A-D are later used in the video analysis in Figs. 3-7.

Fig. 2. An individual frame from video C30. Markers: A) Flow stagnation from the nozzle, covered for filming, B) Cavitation cloud growth, C) Cavitation closure and D) No cavitation. The areas (A-D) are also presented in Fig. 1. 
Fig. 3. Frequency map of $\sigma=0.811$. The $X$ and $Y$-axes present the pixel coordinates in the video.

Fig. 4. Frequency map of $\sigma=0.908$. The $X$ and $Y$-axes present the pixel coordinates in the video.

Fig. 5. Frequency map of $\sigma=1.159$. The $X$ and $Y$-axes present the pixel coordinates in the video.

Fig. 6. Frequency map of $\sigma=1.515$. The $X$ and $Y$-axes present the pixel coordinates in the video.

Fig. 7. Frequency map of $\sigma=1.897$. The $X$ and $Y$-axes present the pixel coordinates in the video.

Fig. 8. AE signal voltage levels for varying cavitation numbers. A) and B) represent fully developed cavitation with cloud shedding, C) represents cavitation that is assumed to be sheet cavitation, and $\mathrm{D}$ ) represents a cavitation number where cavitation no longer occurs.

Fig. 9. The demodulated signal power spectral density compared to that of the original signal.

Fig. 10. Cavitation shedding frequency measured by $A E$ as a function of cavitation number.

Fig. 11. Simulated shedding frequency as a function of cavitation number in the cavitation tunnel by Gavaises et al. (2015). The Strouhal number St was defined as the ratio of shedding frequency multiplied by the gap size of $2.5 \mathrm{~mm}$ and the mean flow velocity at the $25 \mathrm{~mm}$ radius of the diverging channel. Reprinted from International Journal of Multiphase Flow, Vol. 68, M. Gavaises, F. Villa, P. Koukouvinis, M. Marengo, J. Franc, Visualisation and les simulation of cavitation cloud formation and collapse in an axisymmetric geometry, Fig. 15, Copyright 0301-9322/C 2014 Elsevier Ltd, with permission from Elsevier.

Fig. 12. Comparison of the results by Gavaises et al., the video analysis, and the $A E$ measurements, regarding the relation between shedding frequency and cavitation number.

Fig. 13. Cavitation shedding frequency as a function of erosion time, combined with volume loss and erosion depth. Cavitation number $\sigma=0.87$.

\section{Tables}

\begin{tabular}{l|l|l|l|l} 
Video ID & $\begin{array}{l}\text { Cavitation } \\
\text { number } \sigma\end{array}$ & $\begin{array}{l}\text { Upstream } \\
\text { pressure (MPa) }\end{array}$ & $\begin{array}{l}\text { Downstream } \\
\text { pressure (MPa) }\end{array}$ & Length (frames) \\
\hline C27 & 1.897 & 2.909 & 1.905 & 1738 \\
C28 & 1.515 & 3.166 & 1.907 & 1495 \\
C29 & 1.159 & 3.562 & 1.912 & 1356 \\
C30 & 0.908 & 4.027 & 1.916 & 1331 \\
C31 & 0.811 & 4.273 & 1.913 & 2895
\end{tabular}

Table 1. Video lengths and cavitation tunnel operation points. 